\title{
El sistema Braille: Bases para su enseñanza- aprendizaje
}

\section{Cecilia Simón, Esperanza Ochaíta y Juan Antonio Huertas}

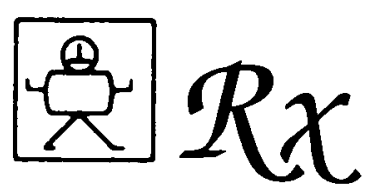

¿Qué es el braille?, ¿cómo leen las personas ciegas?, ¿que peculiaridades tiene la lectura cuando la información se recoge mediante el tacto?, ¿qué dificultades tiene la lectura braille?, ¿qué factores se deben tener en cuenta cuando se quiere enseñar a leer a un niño ciego?. Estas y otras cuestiones son el punto de partida de este artículo.

\section{¿QUE ES EL BRAILLE?}

El sistema de escritura y lectura principalmente empleado por las personas ciegas y por las que tienen deficiencias visuales graves es el braille. Este sistema está diseñado para poder ser explorado de forma táctil y su unidad básica está constituída por la celdilla. Dentro de cada celdilla se pueden situar un conjunto de puntos en relieve en seis posiciones diferentes. Las distintas combinaciones de puntos dan lugar a diferentes letras, pudiéndose así representar todas las letras del alfabeto, los números y también los diferentes signos de puntuación (para una revisión más exhaustiva ver Rosa, Huertas y Simón, 1993).

Este sistema de lecto-escritura, como cualquier otro, tiene una serie de problemas o dificultades que vamos a analizar a continuación. Con ello no queremos presentar un panorama pesimista de las posibilidades que tienen los ciegos para acceder a la información escrita. Pretendemos más bién que los educadores sean conscientes de tales dificultades para poder mejorar los sistemas de instrucción lectora. Podemos clasificar esos problemas en dos grupos: unos derivan del propio sistema de nota-
Figura 1.- Alfabeto braille

\begin{tabular}{|c|c|}
\hline 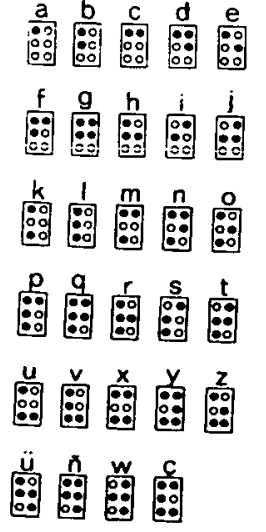 & 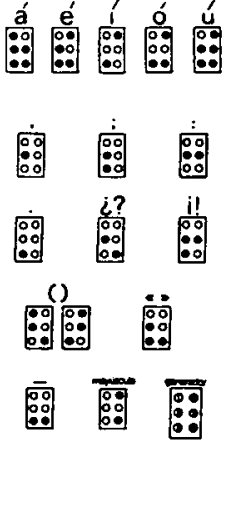 \\
\hline
\end{tabular}

ción escrita, mientras que los segundos tienen que ver con el tacto, el sistema sensorial con que se recoge la información. Analizamos en primer lugar los principales problemas derivados del sistema braille.

Como vemos en la figura 1 , las letras braille están formadas por configuraciones de puntos. El lector puede cometer errores en la identificación de alguno de esos puntos, lo que 


\section{2}

le llevará a reconocer una letra diferente a la que está leyendo, tanto si omite como si añade un punto. Así por ejemplo, si no percibe el punto central de la letra "y» la identificará como una "x». Además, los conjuntos de puntos que forman cada una de las letras tienen una configuración espacial, lo que frecuentemente origina confusiones debidas a la la rotación de esa configuración. De esta forma, se pueden confundir entre sí los grupos de letras $\mathrm{d}-\mathrm{f}-\mathrm{j}-\mathrm{h}$, los formados por n-z-é, o los formados por e-i. Como es lógico estas confusiones pueden llevar al lector a equivocarse en la identificación de una palabra, como por ejemplo, «saco» por «taco», o «jurar» por "durar».

Además, el sistema braille utiliza las mismas configuraciones de puntos para signos diferentes. Los números tienen la misma configuración de puntos que las primeras letras del alfabeto y el lector sólo puede distinguirlos por un signo especial que se pone antes del número. También las letras mayúsculas son iguales las minúsculas, utilizándose una configuración especial que indica la mayúscula. Sin duda, el hecho de utilizar una misma configuración para signos diferentes hace aumentar las dificultades en la discriminación y, en consecuencia, los problemas de aprendizaje del braille.

Otro de los problemas que tiene el braille es que las vocales acentuadas tienen configuraciones de puntos que son completamente diferentes a las de las vocales sin acentuar. Esto supone una dificultad añadida en el aprendizaje de la lecto-escritura para los niños ciegos. Estos niños $\longrightarrow$ los adultos que se inicien en el braille- deben aprender un signo especial para cada una de las vocales acentuadas. Además, como muestra la figura 2 , las vocales acentuadas tienen una configuración bastante compleja y pueden confundirse, por errores de rotación o añadido de puntos, con otras letras del alfabeto.

Por otra parte hay que tener en cuenta que los textos braille, a diferencia de los escritos en tinta, no proporcionan al lector puntos de referencia claros sobre la disposición de las páginas. Nos referimos a la identificación rápida de los márgenes, finales de párrafo, cambios de epígrafe, conceptos calve, etc.., ya que en el sistema braille no es posible utilizar los marcadores que se utilizan en tinta (subrayados, negritas, cambios de letras, cuadros, etc..).
Figura 2.- Vocales acentuadas y sin acentuar

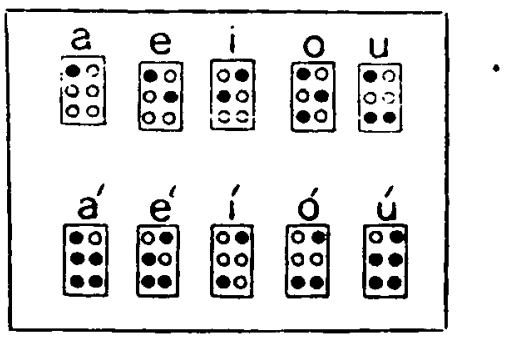

Para terminar señalaremos dos problemas añadidos en la utilización de los textos braille. Una página escrita mediante este sistema ocupa más del doble que una en tinta. Además el material que se utiliza para escribir en relieve (cartulina o plástico) ocupa mayor volumen que el papel normal, lo que da lugar a que los textos sean de gran tamaño, dificultando su manejo y transporte. Además, los textos escritos en cartón -los de plástico originan mayores problemas de fatiga táctil — se degradan si se utilizan repetidamente, dando lugar a problemas de legibilidad con los consecuentes problemas de comprensión y velocidad lectora.

Estos problemas del sistema braille han llevado a diferentes autores a modificar el código para intentar mejorar el rendimiento lector de los invidentes. Podemos clasificar estos intentos de modificación en tres grupos. En primer lugar, se han creado sistemas de braille comprimido o estenotipias, con el objetivo de trasmitir mayor cantidad de información con menor número de caracteres. En segundo lugar, se ha intentado modificar el número de puntos que componen la celdilla braille. Por último, se ha planteado también la posibilidad de diseñar un código de lectura diferente utilizando letras romanas en relieve o un código mixto que combine letras romanas y puntos en relieve.

Comencemos por los sistemas de braille comprimido o estenotipia. Las investigaciones realizadas por Bruteig (1987), Foulke (1982) y Nolan y Kederis (1969) sobre este tema no llegan a resultados totalmente concluyentes. Aunque en muchos casos la utilización de un sistema de braille comprimido aumentaba la velocidad lectora, esto dependía de diferentes factores tales como la familiaridad de las palabras, el efecto del contexto o el nivel lector de los sujetos estudiados.

Por otra parte, Foulke y sus colaboradores (Foulke, 1982; Foulke y Warm, 1967; 
Warm, Clark y Foulke, 1970) han intentado añadir una nueva fila a la celdilla para, con ocho puntos, aumentar el número de combinaciones posibles entre dichos puntos y poder así utilizar configuraciones para contracciones. Los resultados de estos autores parecen indicar que el aumento en el número de puntos no baja la discriminabilidad ni la velocidad lectora.

En fechas recientes se han hecho varios intentos para crear nuevos códigos de lectura táctil. Por ejemplo el «Fishburne Code», elaborado por Shafrach en 1986, dirigido especialmente a los individuos que pierden la visión en la edad adulta. Este sistema utiliza cuatro patrones de símbolos diferentes (puntos, líneas verticales, lineas horizontales y líneas oblicuas) y parece permitir una mayor rapidez en el aprendizaje y una mejor discriminabilidad de los símbolos, aunque no disponemos de estudios experimentales que apoyen tales afirmaciones. Otro sistema de lectura táctil es el elaborado por Thurlow (1988) y conocido por «C5». En él las letras del alfabeto se representan tanto por puntos como por trazos horizontales, verticales y diagonales que simulan el contorno del alfabeto romano. De nuevo, las investigaciones llevadas a cabo con este sistema de lectura no nos proporcionan resultados concluyentes, por lo que de momento, no parece representar una alternativa real al sistema braille.

Pasemos ahora a analizar las dificultades debidas a la urilización del tacto. En primer lugar, como veremos en el apartado siguiente, los ciegos deben identificar los caracteres escritos mediante la exploración realizada con la yema de sus dedos, por lo que la cantidad de información que pueden percibir está limitada a la amplitud perceptiva de éstas —que parece ser la de una celdilla-. Además algunos autores afirman que el sistema táctil es más lento y menos preciso que el visual a la hora de recoger ciertas características de la información, como son las espaciales. (Loomis, 1980; Millar, 1978; Sherrick y Craig, 1982).

Precisamente debido a esas características del tacto, los diferentes expertos en el tema coinciden en señalar que uno de los principales problemas de la lectura braille es su lentitud. Como sabemos, la velocidad media de un lector vidente experto oscila entre las 280 y las 350 palabras por minuto $(\mathrm{p} / \mathrm{m})$. Sin embargo, la velocidad media de los lectores adultos expertos en braille no supera, salvo en casos excepcionales, las $130 \mathrm{p} / \mathrm{m}$. Esta lentitud, sin duda, supone una desventaja considerable para el acceso a la información escrita por parte de las personas invidentes, lo que ha hecho que se busquen sistemas de lectura diferentes. Uno de estos sistemas es el denominado «libro hablado» que consiste en la gravación de texto en cinta magnetofónica. Tales cintas pueden ser escuchadas a velocidades diferentes y constituyen un medio alternativo que permite la lectura y el estudio de forma más rápida, aunque plantea problemas para el repaso.

Debido a los problemas que implica recoger la información escrita con el tacto, se han llevado a cabo distintos trabajos de entrenamiento con la intención de mejorar la rapidez de la discriminación táctil y consiguientemente, la velocidad lectora. Algunos de ellos, en los que los que se pretendía que los lectores identificasen los caracteres escritos, han obtenido resultados contradictorios. Ciertos autores (Nolan y Kederis, 1969; Henderson, 1967) encontraron mejoras en la velocidad lectora de los sujetos mientras que otros (Flanagan y Joslin, 1969; Umstead, 1970; Cates y Sowell, 1990) no obtuvieron mejoras relevantes. Probablemente, los resultados de estos trabajos no sean concluyentes por la falta de control de ciertas variables implicadas en el entrenamiento. Así pensamos que sería útil realizar nuevos trabajos de este tipo que tengan una mayor rigor metodológico.

En cualquier caso es importante señalar que, aunque todos los problemas que hemos citado sean reales y deban ser tenidos en cuenta en la instrucción del braille, este sistema de lectura es un instrumento básico e insustituible para las personas ciegas. Investigaciones realizadas en fecha reciente (Daneman, 1988; Simón, 1993) ponen de manifiesto que las personas que leen en braille alcanzan una comprensión del texto similar a la de los videntes de su misma edad y nivel educativo. Así pués, desde nuestro punto de vista, no existe en la actualidad, un sistema sustitutivo para el braille aunque, como ya hemos dicho, existan otros complementarios. Los esfuerzos educativos deben dirigirse a mejorar este sistema de lectura en la medida de lo posible. Además, como hemos dicho en otra ocasión (Rosa, Huertas y Simón, 1993) los avances en la tecnología —utilización de orde- 
nadores con sistemas periféricos para ciegospermiten cada vez más el intercambio de información entre personas ciegas y videntes.

\section{¿COMO SE UTILIZA EL SISTEMA BRAILLE?}

Las personas ciegas emplean las yemas de los dedos para identificar los caracteres escritos. Pese a que, como comprobó Foulke (1964) todos los dedos de la mano son capaces de recoger información precisa de los caracteres braille, los lectores generalmente se limitan a utilizar los índices, a excepción de los muy expertos que, en ocasiones, también son capaces de recoger información con otros dedos. Autores como Kusajima (1974) han puesto de manifiesto que existen diferencias entre los movimientos de los dedos de los distintos lectores. A medida que éstos van siendo más diestros en el movimiento de las manos por las líneas del texto, se producen cambios en el tipo de exploración que realizan con sus dedos. Los lectores poco diestros, cuando leen una línea, mueven los dedos con lentitud deteniéndose continuamente sobre las letras y ralizando tres tipos de movimientos (horizontales, sagitales y de prensión sobre el papel). Los sujetos con niveles medios de destreza lectora realizan una exploración más rápida y con menos fluctuaciones, mientras que los más diestros llevan a cabo movimientos rápidos, continuos y uniformes de tal manera que, salvo en los repasos, los dedos no realizan fluctuaciones.

También existen diferencias entre los lectores braille en el tipo de movimientos de las manos que realizan para explorar las líneas del texto y para pasar de una línea a otra. A partir de la revisión de las investigaciones que se han realizado sobre el primer tema (Lowenfield y col. 1969; Kusajima, 1974; Davidson y col., 1980; Bertelson y col., 1985; Simón 1994; Simón, Ochaíta y Huertas, en prensa) podemos distinguir los siguientes patrones de movimiento de las manos. 1) Lectura unimanual, en la que sólo se uriliza una mano para explorar el texto: el dedo izquierdo permanece en el comienzo de la línea mientras que el derecho explora el texto. 2) Exploración conjunta, cuando ambos índices exploran juntos la línea, moviéndose uno al lado del otro. 3) Exploración disjunta, en la que los dedos índices realizan movimientos independientes a lo largo de la línea y, por lo tanto, cada mano explora diferentes partes (por ejemplo mientras una mano explora la parte inicial de la línea la otra explora la parte final). 4) Exploración disjunta simultanea, en la que hay un periodo de tiempo durante el que ambas manos leen diferentes partes del texto explorándolo de forma simultanea y, en consecuencia la información es recogida por éstas en paralelo (Bertelson y col.,1985). 5) Exploración mixta que es una combinación de la exploración conjunta y la disjunta. Bertelson y col. (1985) describen este patrón como un movimiento en el que generalmente la mano izquierda lee sola el comienzo de la línea, después ambas manos exploran juntas la parte central hasta que la mano izquierda pasa a la línea siguiente, y la mano derecha termina de leer sola el final de la línea.

Existen también diferentes estrategias para pasar de una línea a otra. En un trabajo reciente (Simón, 1994; hemos encontrado los siguientes. 1) Patrón unimanual, en el que una sóla mano cambia de línea, bien retrocediendo por la línea que ha sido leida, bien bajando a la línea siguiente y retrocediendo por ella hasta su comienzo. 2) Patrón unimanual con indicador, cuando una mano cambia de línea mientras que el dedo índice de la otra, es utilizado como guía en los cambios de línea (se situa al comienzo de cada línea). 3) Patrón bimanualunimanual, de acuerdo con el cual las dos manos se sitúan al final de la línea, una baja primero a la línea siguiente $y$, a continuación lo hace la otra para unirse a la mano que ha bajado primero. 4)Patrón disjunto: antes de que una mano haya finalizado la exploración de una línea la otra se situa en la línea siguiente.

Pero ¿que es lo que determina la utilización de uno u otro patrón de movimiento de las manos y de cambio de linea?, ¿existe alguna relación entre estos patrones y el rendimiento lector?. Según algunos autores (Kusajima, 1974; Bertelson y col. 1985; Rosa y Huertas, 1988; Simón, 1994; Simón, Ochaíta y Huertas, en prensa), existe una correspondencia entre el nivel lector los patrones de movimiento de las manos y cambio de líneas que se utilizan en el braille. Así, los lectores poco expertos llevan a cabo una lectura unimanual; a medida que se adquieren mayores destrezas lectoras se van empleando las dos manos de forma progresiva, hasta llegar al nivel de lectores expertos en el que predomina el patrón disjunto. 
Además, Bertelson y col. (1985), Simón (1994), Simón, Ochaíta y Huertas (en prensa) han encontrado una forma especial de exploración disjunta a la que denominan disjunta simultanea. Como hemos dicho anteriormente, en este tipo de patrón ambas manos leen diferentes partes del texto, pero de forma simultanea (mientras la mano izquierda termina de leer una línea la derecha está leyendo el comienzo de la siguiente), lo que parece implicar que ambas manos recogen la información de forma paralela. Si esto fuera cierto, cabría pensar en la existencia de un ấmacén donde la información dada por ambas manos sería guardada e integrada para dar sentido a lo leído. Aunque la existencia de ese patrón disjunto simultanéo ha sido cuestionada por Millar (1987 y 1989), nosotros hemos obtenido datos experimentales que nos permiten confirmarla. Es importante señalar, por las implicaciones educativas que tiene, el hecho de que son las personas que mueven las manos siguiendo tal parrón, las que alcanzan velocidades lectoras superiores a la media. Así, mientras que la velocidad lectora media de un ciego experto es de $100-120 \mathrm{p} / \mathrm{m}$, la de los lectores que emplean este tipo de patrón llega aumentar hasta 150-165 p/m.

Desde nuestro punto de vista, los resultados obtenidos en estas investigaciones pueden ser utilizados para la instrucción del braille. Como hemos visto, la conducta más eficaz para conseguir una mayor velocidad lectora es aquella en la que se utilizan las dos manos y, más específicamente, el patrón de movimientos que corresponde a la «exploración disjunta». Así pués, proponemos entrenar los movimientos de las manos de los lectores invidentes para faciliar la adquisición de ese patrón de disjunto. Los resultados obtenidos en una investigación reciente (Simón, 1994) ponen de manifiesto que los niños ciegos van utilizando los diferentes patrones anteriormente descritos a medida que avanzan sus niveles escolares. Pero también señalan que dicho progreso se va produciendo a medida que el proceso de lectura se hace más automático. Por tanto pensamos que se debe ir entrenando el movimiento de las manos y el de cambio de línea teniendo en cuenta el nivel lector actual del niño y su zona de desarrollo próximo, es decir, avanzando en la instrucción en el nivel siguiente al que ya tiene el niño. En este sentido hay que tener en cuenta además que no es sólo el movimiento de las manos lo que determina la velocidad lectora, ya que los sujetos que realizan este patrón disjunto son también muy rápidos en la identificación de los caracteres braille.

\section{ENSEÑANZA DE LA LECTURA BRAILLE}

En contraposición con la gran proliferación de trabajos sobre los diferentes aspectos implicados en la enseñanza de la lectura a los niños videntes, existen pocas investigaciones sobre cómo enseñar a leer a las personas ciegas y deficientes visuales. Por ello en la actualidad no existe acuerdo entre los profesores de los niños invidentes sobre el método de enseñanza más adecuado. Lamentablemente, tampoco nosotros podemos dar un imagen coherente y cerrada sobre cúal debe ser el mejor método para la enseñanza del braille. No obstante, en las páginas que siguen, intentaremos unificar todos aquellos factores que consideramos básicos para su instrucción. Comenzaremos revisando los requisitos básicos que los profesores han de tener en cuenta a la hora de iniciar a los niños ciegos en la lectura y, posteriormente, pasaremos a discutir lo que actualmente se sabe sobre métodos de enseñanza explícita del braille para niños. Por último, analizaremos una de las grandes polémicas en el tema de la enseñanza de la lectura a los ciegos y deficientes visuales, que gira en torno a cúal es el código más adecuado para un niño con deficiencia visual.

\section{Factores básicos a tener en cuenta en la instrucción lectora del sistema braille}

Cuando un niño ciego va a iniciar su intrucción en la lectoescritura braille ha de tener - como el vidente - un determinado nivel de desarrollo cognitivo y lingüístico que incluya unas habilidades metalingüísticas concretas. Además es necesario que haya adquirido la sensibilidad tactil y las destrezas motoras manuales adecuadas. Pero también es necesario que tenga interés o motivos para aprender a leer. Puesto que existen muy pocos datos sobre la importancia de todo esto en la adquisición y desarrollo de la lectura braille, no podemos hablar aquí de 
«prerequisitos» en sentido estricto (Maldonado y Sebastian, 1987). Sin embargo pensamos que los profesores de los niños invidentes han de tener en cuenta todos estos factores a la hora de iniciar su instrucción lectora.

Empecemos por analizar el desarrollo cognitivo de los niños ciegos. Las investigaciones realizadas al respecto (para una revisión más exhaustiva remitimos al lector al trabajo de Ochaíta, 1993) ponen de manifiesto que las personas ciegas sin deficiencias asociadas adquieren capacidades cognitivas funcionalmente equivalentes a las de las personas videntes. Pero hay que tener en cuenta que la falta de visión y la consecuente recogida de la información sobre el mundo con otros sistemas sensoriales - principalmente el tacto y la audición- hace que el desarrollo psicológico de los niños ciegos transcurra, a veces, de forma distinta al de los videntes. Cuando la visión falta o está seriamente dañada, el individuo humano ha de construir su desarrollo psicológico utilizando vias alternativas que le permitan compensar, en el sentido vygotskiano del término, su defecto visual (Vygotski, 1927).

Desde luego, el tacto y el oido son menos apropiados que la visión para conocer los objetos y su situación en el espacio. Así las peculiaridades del desarrollo de los ciegos pueden explicarse por el hecho de tener que utilizar estos sistemas sensoriales para conocer el mundo. Los bebés ciegos disponen de mecanismos alternativos para relacionarse con las personas que les rodean pero tienen ciertos problemas o retrasos para construir la permanencia de los objetos. Desde que el niño empieza a hablar, el lenguaje va a constituir para él un sistema muy poderoso para compensar las dificultades originadas por la falta de visión. Así, las primeras imitaciones diferidas y los juegos de ficción que hacen los niños ciegos son, en muchos casos, de caracter verbal.

En el periodo escolar, las peculiaridades del desarrollo cognitivo de los ciegos se ponen claramente de manifiesto cuando analizamos el desarrollo de las operaciones lógicas (Ochaíra 1982 y 1983; Ochaíta, Rosa, Moreno, Pozo y Fernandez 1985; Rosa, Ochaíta, Moreno, Carretero y Pozo 1986). Los niños ciegos tienen la misma competencia lógica que los videntes ya que resuelven las tareas concretas que tienen relación con el lenguaje (clasifica- ciones, seriaciones verbales) a edades similares. Por el contrario, cuando los invidentes tienen que resolver problemas lógicos con materiales de caracter figurativo o espacial (seriaciones y operaciones espaciales) el hecho de tener que recoger la información con el tacto les plantea dificultades que les hacen estar retrasados respecto a los videntes.

Pero el papel del lenguaje -interiorizado en forma de pensamiento verbal o hipotético deductivo - parece remediar o compensar, al llegar a la adolescencia, los problemas figurativos derivados de la falta de isión. Entre los 12 y 15 años de edad los ciegos resuelven sin problemas las tareas lógicas espaciales y figurativas, aunque llegan a hacerlo siguiendo rutas diferentes a las de los videntes. Mientras que estos últimos resuelven estas tareas de forma realmente concreta, llevando a cabo acciones y manipulaciones de los objetos reales, los ciegos tienen que acudir a la mediación que les proporciona el lenguaje, lo que supone una vía alternativa bastante compleja. Por todo ello, las investigaciones realizadas sobre las competencias lógicas de los adolescentes en el periodo piagetiano de las operaciones formales, no han encontrado diferencias entre ciegos y videntes.

Volvamos ahora al tema de la instrucción lectora. Todo lo hasta ahora dicho nos permite afirmar que las capacidades intelectuales de los niños ciegos son funcionalemnte equivalentes a las de los videntes. Por ello pensamos que ambos deben empezar el aprendizaje lector a edades semejantes: en el inicio de la E.G.B. Sin embargo, no podemos esperar que dicho aprendizaje transcurra en unos y otros de manera idéntica. Como dijimos al comienzo de este artículo el sistema braille parece tener más dificultades que el visual, tanto por los problemas inherentes al propio código, como por las que conlleva la utilización del tacto. Los niños deficientes visuales han de aprender un sistema formado por configuraciones espaciales de puntos recogiendo la información con el tacto, por lo que el acceso inicial a la información escrita les resulta más difícil que a los videntes. Las investigaciones realizadas sobre la adquisición de la lectura en los invidentes ponen de manifiesto estas dificultades (Equipo UAM/ONCE, 1989 y Simón, 1994). Por ello pensamos que profesores e investigadores deben trabajar juntos para diseñar un buen 
método de instrucción que permita a los niños ciegos acceder a la información escrita de la manera más fácil y rápida que sea posible.

Por otro lado, como sabemos, todo sistema de lectura tiene un componente verbal que, como hemos visto no solo no presenta problemas en el niño ciego, sino que le permite remediar los problemas originados por la ausencia de visión. A continuación resumimos al lector aquellos aspectos del desarrollo del lenguaje que tienen mayor relación con la instrucción lectora.

Los investigaciones realizadas con niños videntes muestran que el nivel de desarrollo lingüístico que tenga un niño es muy importante en el aprendizaje de la lectura. Indudablemente, el niño comprenderá mejor un texto y, probablemente lo leerá con mayor rapidez, si conoce las palabras escritas en el mismo y la gramática de su lengua. Así es importante que cuando los niños aprendan a leer tengan un vocabulario adecuado a su edad, una pronunciación correcta, una buena comprensión y expresión verbal. Como más tarde veremos, también es muy importante que hayan adquirido ciertas habilidades metalingüísticas que les permitan reflexionar sobre el propio lenguaje.

La bibliografía existente sebre el desarrollo del lenguaje en los niños ciegos permite afirmar de forma clara que no existen problemas especiales en este área cuando falta la visión (para una revisión exhaustiva ver Ochaíta, 1993). Estos niños tienen un desarrollo fonológico normal, adquieren sus primeras palabras en el mismo rango de edad que los videntes y el desarrollo posterior de su léxico puede considerarse tambien normal tanto desde el punto de vista cuantitativo como cualitativo, aunque tenga ciertas peculiaridades. Por ejemplo los niños invidentes utilizan antes las palabras referidas a los objetos y acciones que pueden percibir directamente, que aquellas otras que se refieren a objetos y acciones lejanos. También el desarrollo gramatical de estos niños es perfectamente equiparable al de los videntes. Desde el punto de vista comunicativo, la ausencia de visión produce ciertas peculiaridades en el adquisición del lenguaje. Así los niños invidentes tienen ciertos retrasos en la adquisición de los términos deícticos, como por ejemplo los pronombres personales "yo» y «tu» o los adverbios "aquí» "allí». Estos problemas son superados normalmente en etapas previas a la del inicio de la instrucción lectora por lo que no plantean problemas especiales para esa instrucción.

Pasemos ahora a analizar nuestros conocimientos sobre las babilidades metalingüisticas. Desde hace varios años las investigaciones realizadas con niños videntes han destacado la importancia de estas habilidades -que, en general, implican capacidad de pensar sobre el lenguaje y operar con el-y más concretamente de las habilidades fonológicas en la adquisición y desarrollo de la lectura (Sebastian y Maldonado, 1986; Maldonado y Sebastian, 1987; Maldonado, 1990; Alegria y Morais, 1991; Mann, 1991; Tunmer, 1991). Autores tales como Maldonado y Sebastian (1987) han demostrado que constituyen prerequisitos para aprender a leer. Otros autores (para una revisión ver Brady y Shankweiler, 1991; Rueda, 1993) concluyen, además, que esas habilidades pueden entrenarse no sólo para facilitar el aprendizaje de la lectura sino tambien para remediar problemas lectores ya existentes.

El papel de estas capacidades metalingüísticas en el aprendizaje de la lectura ha sido puesto de manifiesto tambien en los niños sordos (Alegria y Leybaert, 1987; Asensio, 1989). Sin embargo carecemos de investigaciones que estudien sus relaciones con el aprendizaje de la lectura en los niños ciegos y deficientes visuales. En cualquier caso, dada la importancia que las capacidades metalinguísticas han demostrado tener en la lectura, pensamos que deben tenerse en cuenta en los métodos instruccionales del braille, aunque se necesiten nuevas investigaciones en este área.

Pasemos ahora a analizar aquellos aspectos relacionados con el tacto que pueden ser importantes a la hora de enseñar a leer braille: el entrenamiento de la sensibilidad tactil y de las destrezas motoras de las manos.

En primer lugar, debemos tener en cuenta que la capacidad de discriminación perceptiva que el niño tenga le va a permitir identificar con mayor rápidez los caracteres escritos. Los trabajos realizados sobre percepción táctil han demostrado que, el hecho de carecer de visión, no lleva consigo una mayor sensibilidad cutanea. Sin embargo, la práctica en el uso del tacto que la ceguera conlleva sí facilita la capacidad de discriminación mediante este sistema sensorial (ver Blanco y Rubio, 1993). En este sen- 


\section{8}

tido puede ser importante incluir ejercicios de discriminación táctil en los en las etapas previas a la instrucción específica del braille. Tales ejercicios pueden consistir en discriminación de formas de complejidad creciente, reconocimiento de figuras, reconocimiento de configuraciones de puntos, etc...

Además, como ya sabe el lector, el movimiento de los dedos y de las manos es muy importante en la lectura braille. Así es importante entrenar las destrezas manuales en los diferentes momentos de la instrucción lectora. En las etapas iniciales deben llevarse a cabo ejercicios de coordinación bimanual (ensartado de cuentas, ejercicios con tableros de clavijas, rompecabezas, etc..), de independencia sagital, de movimiento de muñecas, de búsqueda de símbolos distribuidos de izquierda a derecha a lo largo del texto, etc.. Posteriormente, el entrenamiento debe dirigirse al movimiento de las manos por las líneas del texto y al cambio de líneas, tal como dijimos en páginas anteriores de este mismo artículo.

Para terminar este apartado, hemos de señalar que el niño ciego debe estar interesado en aprender a leer. Generalmente los niños videntes tienen un gran interés en el comienzo de la iṇstrucción lectora y este interés es debido a que estan familiarizados desde edades muy tempranas con los materiales escritos, por ejemplo carteles publicitarios, libros, cuentos, etc. De esta forma, y gracias a la intervención del adulto que les lee estos carteles o los cuentos, el niño se va haciendo cada vez más consciente de que los caracteres impresos «quieren decir algo», «tienen algún significado». Así, los niños van tomando conciencia del propósito de la lectura, despertándose en ellos una motivación clara por aprender a leer.

Este proceso no se produce tan fácilmente en los niños ciegos. La gran mayoría de estos niños son hijos de padres videntes, por lo que normalemente no tienen un contacto temprano con el tipo de materiales que van a utilizar realmente para leer. Por ello, puede resultarles poco motivante aprender a leer en braille. Recordemos que para que una actividad resulte motivante para un niño tiene primero que ser relevante para él, tiene que estar dirigida a una meta, a un propósito consciente, autoatribuido y que entronque con alguno de sus intereses básicos (leer cuentos, aprender cosas nue- vas, etc..). Resulta entonces necesario que las personas que rodean al niño le destaquen, en la práctica cotidiana, las funciones y la relevancia que, para sus propios intereses, tiene el acceso a lo escrito. Para ello es conveniente captar su atención con materiales atractivos (por ejemplo libros con diferentes texturas y asociados con sonidos) que les hagan comprender el mundo de significados que guarda un libro.

Así pues, se hace imprescindible que los profesores incorporen la motivación por la lectura como un elemento más del curriculo, que siguiendo la nueva reforma educativa, debería esta contemplado dentro del contenido referido a «actitudes, normas y valores». En definitiva, desde antes del inicio de la instrucción lectora propiamente dicha, tanto los padres como el profesor deberan hacer consciente al niño el propósito de la lectura y de su utilidad como instrumento de aprendizaje y comunicación.

\section{Métodos de instrucción braille}

Los métodos más conocidos para de enseñanza-aprendizaje del braille son los desarrollados en lengua inglesa, concretamente los «Basal reader» y dentro de estos el llamado «Patterns». Este método ha sido creado para instruir a los ciegos desde las primeras etapas de la lectura braille. Al final del programa se introducen las contracciones y abreviaturas del braille tipo II. Recientemente se le ha añadido el «Patterns Prebraille Program» que introduce conceptos previos y habilidades linguíísticas necesarias para la lectura. Una de las principales ventajas de este método es que está basado en un análisis lingüístico del braille de tal manera que las palabras se van introducciendo de acuerdo su dificultad.

En lengua castellana no disponemos de métodos tan elaborados para la enseñanza del braille, aunque en la actualidad existan algunos interesantes. Entre los más recientes y conocidos se encuentra el llamado "Tomillo" descrito por Lucerga en 1990. Este método se ha elaborado de acuerdo con los siguientes criterios: a) En primer lugar se trata de un método fonético que apoya la presentación de contenidos significativos al mismo tiempo que respeta las peculiaridades de la exploración táctil; b) Además, se presentan los contenidos significativos adecuados a la edad de los niños, para lo que, durante las primeras estapas del aprendi- 
zaje del braille, se utilizan palabras y frases con estructuras lingüísticas familiares para el niño, cargadas además de contenido emocional; c) Se emplean materiales atractivos que estimulen el deseo de leer, como por ejemplo volúmenes reducidos y manejables para los niños, representaciones en relieve de objetos familiares a los que hagan referencia los textos, cuadernillos de ejercicios o fichas que permitan al niño una participación más activa, etc; d) Se secuencia la presentación de las letras braille teniendo en cuenta las dificultades específicas del sistema (reversibilidad, similitud de signos, dificultades de percepción propias de cada grafe$\mathrm{ma}$ ), las características fonéticas y ortográficas de la lengua y la ordenación utilizada en la enseñanza de la lectura en tinta a los niños videntes (aunque, según Lucerga, aunque este último aspecto deba posponererse a los dos anteriores, también tiene una gran importancia en el caso de los niños integrados en la escuela ordinaria).

Como reconoce Lucerga (1990), aún no se ha realizado un estudio riguroso que permita obtener datos objetivos sobre la eficacia del método mediante la comparación con otros alternativos. No obstante, nosotros consideramos que, a pesar de que los criterios seguidos en la elaboración de éste método son bastante aceptables, debe continuarse trabajando en su perfeccionamiento.

Desde nuestro punto de vista, los profesores no deben intentar enseñar a leer braille basándose simplemente en la traslación a este sistema de los métodos y libros diseñados inicialmente para la instrucción de los niños videntes. Es imprescindible hacer adaptaciones y cambios en el contenido de la metodología instruccional utilizada habitualmente con los videntes. Así , antes de comenzar la instrucción lectora debe evaluarse la situación del niño los factores generales que anteriormente hemos considerado (motivación, lenguaje, destrezas motoras finas y gruesas y sensibilidad táctil). En los primeros momentos de enseñanza del braille se pueden utilizar celdillas de mayor tamaño para facilitar la discriminación entre unas letras y otras. Posteriormente, para la instrucción lectora propiamente dicha, deben tenerse en cuenta las dificultades inherentes al código braille que estudiamos al principio de este capítulo. Así pues, es de especial interés tener en cuenta las confusiones que aparecen en palabras con letras de formas espaciales relacionadas o rotadas (d-f-j-h, n-z-é, e-i, á-u y ó-u), las diferencias de notación entre vocales acentuadas y no acentuadas, o el uso de las mismas configuraciones para letras que para números. En este sentido consideramos que hay que ser extremadamente cuidadoso en la secuencia de enseñanza de los diferentes caracteres braille, secuencia que debe adaptarse a las propias dificultades del sistema y a las confusiones que se producen entre las diferentes configuraciones. Así pues, creemos que debe hacerse un esfuerzo en determinar de forma objetiva y empírica la secuencia óptima de presentación de los caracteres braille para la instrucción lectora.

Sin embargo, no podemos elaborar un método de enseñanza de la lectura estandarizado que sea igual para todas las edades. Obviamente, no es lo mismo enseñar a leer a un niño preescolar que a uno con 14 años. No podemos olvidar que la población que necesita aprender braille puede tener edades muy distintas. Así se han elaborado en lengua inglesa métodos específicos de enseñanza para sujetos de diferentes edades (Harrison, 1987). En relación con lo que se dijo al hablar del codigo braille es importante que los textos sean adecuados al nivel educativo del niño y que tengan una organización y estructura que facilite el acceso al significado de lo escrito. Por ejemplo como ha demostrado Simón (1994), al igual que en la lectura de los videntes, la estructura de los textos (estructura narrativa vs. expositiva) es un factor importante en el recuerdo de los mismos. También Simón (1994) y Simón, Ochaíta y Huertas (en prensa) han mostrado la utilización de diferentes estrategias de reconocimiento de palabras braille (vía fonológica y vía indirecto-inferencial) en función de las características de éstas (como la familiaridad y longitud). Por tanto se recomienda que se emplee en los textos organizadores previos, títulos y encabezamientos claros, listas de palabras clave, resúmenes, etc.

¿Qué código enseñar a los deficientes visuales?

Uno de los grandes problemas con que se encuentran los profesionales dedicados directa $o$ indirectamente a la enseñanza de la 
lectura braille a los deficientes visuales, es qué tipo de código (tinta o braille) debe enseñarse a estos niños. La ausencia de criterios claros ha originado confusiones entre estos profesionales con los consiguientes retrasos lectores en los niños por falta de actuaciones claras.

El proceso que ha de seguirse para determinar qué método de lecrura es más adecuado para los deficientes visuales puede dividirse según Koening y Holbrook $(1989,1991)$ en dos fases. En la primera fase la tarea consiste realizar una primera decisión respecto al tipo de código que aprenderá el niño. Esta decisión debe ser tomada por un equipo multiprofesional. Este equipo tendrá que decidir si el estudiante deberá aprender el código en tinta o el código en braille, o si deberá aprender ambos códigos con igual énfasis. Para tomar este tipo de decisiones será necesario evaluar y considerar los siguientes factores: la visión funcional del niño, la eficiencia y potencial táctil, la evolución de la deficiencia visual, la presencia de problemas asociados, etc... En la segunda fase, el equipo multiprofesional deberá llevar a cabo un seguimiento del niño, revisando la adecuación de la decisión tomada en la primera fase y estudiando la posibilidad de modificarla una vez obtenida la información de la evolución y rendimiento del niño. Por ejemplo, si en un primer momento se decidió enseñar al niño un sólo código, el equipo multiprofesional puede considerar adecuada la inclusión de un segundo código.

Frente a las concepciones tradicionales sobre el uso de los restos visuales en los sujetos que los tienen, actualmente se piensa que, en la medida de lo posible, se deberá intentar el desarrollo, potenciación y uso de la visión (Newman, Hall, Ramseur, Foster, Goldson, Decamp y Grambe, 1982). Pero además se considera que los niños con deficiencias visuales deberán aprender a utilizar otros sentidos, incluyendo el táctil, por si fuera necesario para la lecrura braille.

Además del seguimiento de estas fases, otro factor que se debería tener en cuenta cuando se trata de enseñar el braille a sujetos con restos visuales, es la motivación de estos hacia el aprendizaje de este sistema de lectura. Pueden incidir múltiples factores en la acepración de la enseñanza del braille. Entre estos factores
Holbrook y Koening (1992) desatacan la aceptación de la deficiencia visual, la concepción que se tenga del sistema braille, y la implicación de los padres.

Comencemos con el tema de la aceptación de la deficiencia visual. No es fácil aceptar la pérdida de visión de un hijo, pero tan difícil como eso es la aceptación por parte del propio sujeto de su pérdida de visión. Cuando un equipo multiprofesional considera adecuado que se le enseñe a un niño el código braille pese a tener restos visuales, suele producirse un gran rechazo hacia este sistema de lectura tanto por parte de los padres como del propio sujeto. La razón es obvia: el braille es poco conocido por la población vidente y, cuando se conoce, generalmente está asociado con ceguera total, lo que hace más evidente la gravedad de la deficiencia visual del niño que ha de aprenderlo.

Todo ello hace necesaria la realización de una completa labor terapeutica y de información tanto con los padres como con los propios niños deficientes visuales. Es importante que tanto unos como otros conozcan los aspectos clínicos y funcionales que caracterizan al deficiente visual. Algunos profesionales recomiendan que los padres sepan evaluar la visión funcional de sus hijos, lo que les permitirá tener una mayor conciencia no tanto de lo que su hijo «no tiene» como del resto de visión funcional de que dispone.

Respecto a la idea sobre lo que es el sistema braille es importante que el niño y sus padres acepten el braille como un medio de aprendizaje y no tanto como un símbolo de ceguera. Es importante mostrarles la utilidad de este sistema de lecto-escritura no solo para el aprendizaje escolar, sino también como medio de comunicación. También es interesante que conozcan la posibilidad del uso de otros instrumentos («libro hablado», "cued speak", etc.) que pueden ser de gran urilidad como ayuda a la lectura.

Por último, otro factor que incide sobre la predisposición para el aprendizaje del braille es la implicación de los padres en el proceso. Ya hemos hecho referencia a la importancia que tiene el que éstos conozcan y acepten la limitación visual de sus hijos, pero además, es de gran valor su participación activa en la enseñanza del código braille. 


\section{Referencias}

ALEGRfA, J., y LeYBAERT, J. (1987). Adquisición de la lectura en el niño sordo: un enfoque psicolingüístico. M.E.C. Serie Documentos, 5 .

ALEGRfA, J., y MORAIS, J. (1991). Segmental analysis and reading acqusition. En L. Rieben y Ch. Perfetti (eds.), Leaming to read: Basic reseanch and its implications. Hillsadale, N.J.: LEA.

ASENSIO, (1989). Los proceses de lectura en los deficientes auditivos. Tesis Doctoral no publicada.

Ber Telson, O.; MOUSTY, PH., y D'Aumonte, G. (1985). A study of braille reading: 2. Patterns of hand activity in one-handed and two-handed reading. The Quaterly Journal of Expeimental Psychology, 37A, 235-256.

Blanco, F., y RUBio, M. E. (1993). Percepción sin visión. En A. Rosa y E. Ochaita (eds.), Psicologia cognitiva de la ceguera. Madrid: Alianza Psicología.

BRADEY, S. A., y SHANKWERER, D. P. (1991). Phonological processes in literacy. Hillsdale, N.J.; LEA:

CATES, D. L., y SOWEL, V. M. (1990). Using a braille tachistocope to improve braille reading speed. Journal of Visual Imapirment and Blindness, Dec., 556-559.

DANEMAN, M. (1988). How reading braille is both like and unlike reading print. Memory and Cognition, 16, 497-504.

DAvidson, P. W.; WIIES-Kettenmann, M.; HABer, R. N., y APEUE, S. (1980). Relationship between hand movement, reading comperence and passage difficulty in braille reading. Neuropsychologia, 18, 629-635.

EQUIPO UAM-ONCE (1989). Fundamento y diseño de un proyecto de evaluación de la integración educariva de los niños con necesidades educativas especiales por su deficiencia visual. Integración, 3, 15-21.

FLANINGAN, P. J., y JosLIN, E. (1969). Patterns of response in the perception of braille configuration. New Outlook for the Blind, 63, 232-244.

FOUKK, E. (1964). Transfer of a complex perceptual skill. Perceptual and Motor Skills, 18, 733-740.

FOULKE, E. (1982). Reading braille. En W. Schiff y E. Foulke (eds), Tactual Perception: A Sourcebook. Cambridge University Press.

FOULKE, E., y W ARM, J. (1967). Effects of complexity and redundancy on the tactual recognition of metric figures. Perceptual and Motors Skills, 25, 177-187.

HARRISON, F. (1987). Teaching braille to latecomers in the 12-16 age range. British Journal of Visual Impaiment, 5, 45, 47-49.

Henderson, F. M. (1967). The effect of character resognition training on braille reading. Tesis doctoral no publicada, George Peabody College for Teachers, Nashville.

HoLBROOK, M. C., y KOENING, A. J. (1992). Teaching braille reading to students with low vision. Joumal of Visual Impaiment and Blindness, January, $44-48$.

KOENING, A. J., y HoLBROOK, M. C. (1989). Determining the reading medium for students with visual impairments: a diagnostic teaching approach. Joumal of Visual Imapiment E Blindness, June, 296-302.

KoENING, A. J., y HOLBROoK, M. C. (1991). Determining the reading medium for students with visual impairments: A diagnostic reaching approach. Journal of Visual Imapirment \& Blindness, 83, 296-302.

KUSAJIMA, T. (1974). Visual reading and braille reading: an experimental imestigation of the physiology and psychology of the visual and tactual reading. American Foundation for the Blind.

LOOMIS, J. M. (1980). On the tangibility of letters and braille. Perreption and Psychophysiss, 29(1), $37-46$.

LOWENFELD, B; ABEL, G., y HATLEN, P. L (1969). Blind children learn to read. Springfield, III., Thomas.

LUCERGA, R. R. (1990). La iniciación de los niños deficientes vistuales a la lectura braille. Conferencia Internacional sobre el Braille. Madrid: ONCE.

Maunonado. A. (1990). El desarrollo de la lectura durante los primeros años de escolaridad. Tesis doctoral no publicada. Universidad Autónoma de Madrid.

Maldonado, A., y Sebastián, E. (1985). ¿Existen realmente niños disléxicos?. El País. Suplemento de Educación, 12 de Marzo.

MALDONADO, A., y SEBASTIÍN, E. (1987). La segmentación de palabras: un prerrequisito del aprendizaje de la lectura. Boletín del ICE de la UAM, 9, 1-14.

MANN, V. (1991). Phonological abilities: Effective predictors of future reading ability. En L. Rieben y Ch. Perfetti (eds.), Learning to read: Basic research and its implications. Hillsadale, N.J.: LEA.

MmLAR, S. (1978). Aspects of memory for information from touch and movement. En G. Gordon (ed), Active Touch. Oxford: Pergamon Press.

MILIAR, S. (1987). The perceptual «window» in two-handed braille: Do the left and rigth hands process text simultaneously? Cortext, 23(1), 111-122.

MiLAR, S. (1989). Simultaneos reading with the two hands: Reply to Bertelson and Mousty. Cortex, 25(3), 499-502.

Newman, S. E.; Hall, A.D.; Ramseur, C. J.; Foster, D. J.; Goldson, D. B.; DeCamp, B. L., y Granbe, (1982). Factors affecting the learning of braille.Journal of Visual Imapirment $\&$ Blindness, Feb.

NOLAN, Y. C., y KEDERIs, J. C. (1969). Perreptual factors in braille uord reognition. American Foundation for the Blind Research series, $n^{\circ} 20$.

OCHAf́n, E. (1982). El conocimiento del espacio en los niños ciegos. Tesis Doctoral. Facultad de Filosofía y Letras. Universidad Autonoma de Madrid.

Ochuft八, E. (1983). La teoría de Piaget sobre el desarrollo del conocimiento espacial. Estudios de Psicologia, 14-15, 13-18.

OChaftA, E. (1993). Ceguera y desarrollo psicológico. En A. Rosa y E. Ochaita (eds.), Psicologia cognitiva de la ceguera. Madrid: Alianza Psicología.

OChafta, E.; Rosa, A.; Pozo, J. I., y Fernández-Lagunila, E. (1985). Clasificaciones y seriaciones: un importante "desfase" en el desarrollo cognitivo de los niños ciegos. Revista de Psicología General y Aplicada, 40(3), 395-419.

Rosa, A., y Huertas, J. A. (1988). Peculiaridades de la lectura táctil del braille. Un estudio empírico. Infancia y Aprendizaje, 41, $79-94$. 
Rosa, A.; Huertas, J. A., y Simón, C. (1993). La lectura braille. En A. Rosa y E. Ochaita (eds.), Psicología cognitiva de la ceguera. Madrid: Alianza Psicología.

Rosa, A.; Ochaíta, E.; Moreno, E.; Fernández-Lagunila, E.; Carretero, M., y Pozo, J. I. (1986). Aspectos cognitivos del desarrollo psicológico de los ciegos. Madrid: CIDE.

RUEDA, M. (1993). Conacimiento segmental y dislexia: efectos de la instrucción en la adquisición y mantenimiento del conacimiento fonémico en niños disléxicos. Universidad de Salamanca. Tesis doctoral no publicada.

Sebastiá, E., y MaLdonado, A. (1986). El desarrollo de las estrategias de segmentación de palabras en lectores jóvenes. En J. Meisel (ed.), Adqusición del lenguaje. Frankfurr: Klaus-Dieter Verlag.

SHAFRATH, M. R. (1986). An alternative to braille labeling. Joumal of Visual Impaimment E Blindness, Nov., 955-956.

SIMÓN, C. (1994). El desarrollo de los pracesos básicos en la lectura braille. Madrid: Organización Nacional de Ciegos.

Simón, C.; OCHAfTA, E., y HUERTAS, J. A. (en prensa). Los procesos de reconocimiento de palabras en la lectura táctil del braille. Infancia y Aprendizaje.

Simón, C.; OCHAfTA, E., y HuERTAS, J. A. (en prensa). A specific type of braille reading strategies: the exploration of text by the hands. Chaiers of Cognitive Psychology.

THurLow, W. R. (1988). An alternative to Braille. Joumal of Visual Imapirment E Blindness. 82, 9, 378.

Tunmer, W. (1991). Phonological Awareness and Literacy Acquisition. En L. Rieben y Ch. Perfetti (eds.), Learning to read: Basic research and its implications. Hillsadale, N.J.: LEA.

UMSTEAD, R. G. (1970). Improvement of braille reading through cade recognition training. Tesis doctoral no publicada, George Peabody College for Teachers, Nashville.

VyGoTsKi, L. S. (1927). Defecto y Compensación. En Obras Escogidas, vol V. Problemas de Psicología Anormal y Discapacidades para el Aprendizaje: Fundamentos de Defectología. Moscú: Academia de Ciencias Pedagógicas.

WARM, J.; CLARK, J. L., y FoULKE, E. (1970). Effects of differential spatial orientation on tactual pattern recognition. Pereptual and Motor Skills, 31, 87-94.

\section{El sistema Braille: bases para su enseñanza-aprendizaje Cecilia Simón, Esperanza Ochaíta y Juan Antonio Huertas CL\&E, 1995, 28, pp. 91-102}

Resumen: El objetivo de este trabajo es ofrecer una panorámica actualizada del sistema de lector-escritura empleado por los ciegos y personas con deficiencia visual grave: el braille.

Se presenta una descripción general de este sistema de notación donde se señalan tanto sus características y limitaciones como las principales vias para superar estas limitaciones. También se presenta al lector una revisión crítica de los métodos más empleados para la enseñanza del braille, así como de las aportaciones realizadas por diversas investigaciones para mejorar estos métodos. Igualmente se aborda el problema de la enseñanza de la lectura a los deficientres visuales, concretamente se plantean las decisiones que se deben tomar en relación a qué código enseñar (braille o tinta) a aquellos sujetos que tienen restos visuales.

Así mismo, se hace una reflexión de los principales aspectos que deberían tenerse en cuenta en la instrucción del braille para facilitar su aprendizaje.

Datos sobre los autores: Cecilia Simón es Profesora Asociada del departamento de Psicología Evolutiva y de la Educación de la Universidad Autónoma de Madrid.

Esperanza Ochaíta es Profesora Titular del departamento de Psicología Evolutiva y de la Educación de la Universidad Autónoma de Madrid.

Juan Antonio Huertas es Profesor Titular del departamento de Psicología Básica de la Universidad Autónoma de Madrid.

Los autores trabajan en la actualidad en temas relacionados con la adquisición y desarrollo de diferentes actividades psicológicas en personas con y sin deficiencias asociadas.

Dirección: La dirección de los dos primeros autores es Departamento de Psicología Evolutiva y de la Educación. Facultad de Psicología. Uiversidad Autónoma de Madrid. Cantoblanco, 28049. (Fax. 39752 15) email:csimon@ccuam3.sdi.uam.es.

La dirección del tercer autor es Departamento de Psicología Básica. Facultad de Psicología. Universidad Autónoma de Madrid. Cantoblaco, 28049. (Fax. 39752 15).

(c) PERMISOS PARA CITAR O REPRODUCIR EN OTRAS FUENTES: Se pueden citar libremente hasta 500 palabras. Para reproducir una porción de texto mayor, figuras o ilustraciones, se deberá pedir permiso por escrito a la revista, especificando el uso al que se destina el texto. En todos los casos, se deberá citar el copyright de $C L \& E$. En el caso de artículos o textos que hayan sido a su vez reproducidos en $C L \& E$ los interesados deberán dirigirse tanto a los detentadores del copyright original como a $C L \& E$, en el caso de que se quiera hacer uso de la traducción. FOTOCOPIAS: Para todo lo relacionado con el uso mediante fotocopia del material de esta revista, deberán dirigirse a: CEDRO, C/ José Marañón, 10, 3. ${ }^{\circ}$ Izda. Tel. 59415 75. Fax 4453567 